\title{
Adhesion of platelets to chemotactically responsive and non-responsive neutrophils
}

\author{
K B Pastakia, N E Brownson, D A Terle, L Harvath
}

\begin{abstract}
Aims-To investigate the heterotypic adhesion of unactivated platelets to chemotactically responsive (migrated) and non-responsive (non-migrated) polymorphonuclear neutrophils (PMN).

Methods-Platelets and PMN were isolated from autologous, normal blood. Migrated and non-migrated PMN were separated after $\mathbf{N}$-formylmethionyl-leucylphenylalanine (FMLP) stimulation. Platelets were labelled with a fluorescent monoclonal antibody directed against CD41 (GPIIb-IIIa). Platelets $\left(3 \times 10^{8} / \mathrm{ml}\right)$ and PMN $\left(3 \times 10^{6} / \mathrm{ml}\right)$ were incubated together. Heterotypic cell adhesion was measured in isolated PMN and PMN co-incubated with platelets by flow cytometric analysis of platelet marker fluorescence in PMN gated events. Platelet-PMN adhesion was also visualised by fluorescence microscopy.

Results-In studies of isolated PMN, contaminating platelets were bound to $16-34 \%$ of unstimulated PMN, 7-22\% of stimulated PMN, 2-4\% of migrated PMN, and 17-24\% of non-migrated PMN. When platelets were co-incubated with migrated or nonmigrated PMN, 15-78\% of PMN bound one or two platelets.

Conclusions-Unactivated platelets adhere to isolated PMN in vitro. Fewer unactivated platelets were adhered to migrated PMN than to non-migrated PMN in isolated PMN preparations. These results indicate that platelets adhering to PMN are removed during PMN migration. (f Clin Pathol: Mol Pathol 1996;49:M17-M22)
\end{abstract}

Keywords: platelets, neutrophils, migrate.

Laboratory of Cellular Hematology, Division of Hematology, Center for Biologic

Evaluation and

Research, Food and

Drug Administration, Bethesda, Maryland, USA

K B Pastakia

N E Brownson

D A Terle

L Harvath

Correspondence to: K B Pastakia, $\mathrm{PhD}$

Laboratory of Cellular Hematology, Division of Hematology, CBER, Food and Drug Administration, and Drug Administration, Building 29, Room 329,

Bethesda, MD 20892, USA.

Accepted for publication 31 October 1995

Platelet-polymorphonuclear neutrophil (PMN) adhesion is a dynamic process which contributes to haemostasis and inflammation. In vitro studies show that platelet-PMN interactions have stimulatory and inhibitory effects on the function of both cell types. Cell-cell contact as well as soluble inhibitors seem to be involved in platelet-PMN interactions, ${ }^{12}$ but the mechanisms of these heterotypic interactions have yet to be elucidated. Activated platelet adhesion to $P M N$ is calcium dependent and is mediated by P-selectin (CD62P) $)^{3-5}$ expressed on the activated platelet surface binding to the leucocyte $P$-selectin glycoprotein ligand$1 .^{6}$ Unactivated platelets also bind to PMN when platelets and PMN are incubated to- gether. ${ }^{7}$ However, the binding is independent of divalent cations and may be mediated by a platelet receptor other than P-selectin. ${ }^{7}$

PMN exhibit heterogeneous chemotactic responsiveness in vitro. One subpopulation migrates rapidly in response to a chemoattractant gradient, whereas the other subpopulation fails to migrate. ${ }^{89}$ Cell surface adhesion molecule expression is downregulated differentially on migrated PMN. ${ }^{10}$ In the present study we have characterised the adhesion of unactivated platelets to migrated and non-migrated PMN by flow cytometry and obtained supportive microscopic evidence of platelet-PMN interaction.

\section{Methods}

PLATELET PREPARATION

Platelets were isolated from normal blood collected in anticoagulant citrate dextrose solution ( $75 \mathrm{mM}$ trisodium citrate, $38 \mathrm{mM}$ citric acid, $124 \mathrm{mM}$ dextrose). To obtain platelet rich plasma, blood was centrifuged at $160 \times g$ for 20 minutes at room temperature $\left(20-24^{\circ} \mathrm{C}\right)$. Washed, unactivated platelets were obtained from platelet rich plasma by being centrifuged at $1000 \times g$ for 10 minutes and suspended at a density of $3 \times 10^{8} / \mathrm{ml}$ in Tyrode's/HEPES buffer (TH; $137.0 \mathrm{mM} \mathrm{NaCl}, 2.7 \mathrm{mM} \mathrm{KCl}, 0.42 \mathrm{mM}$ $\mathrm{NaH}_{2} \mathrm{PO}_{4}, \quad 12.5 \mathrm{mM} \quad \mathrm{NaHCO}_{3}, \quad 2.0 \mathrm{mM}$ $\mathrm{MgCl}_{2}, 5.5 \mathrm{mM}$ D-glucose, 5.0 mM HEPES, and $3.5 \mathrm{mg} / \mathrm{ml}$ bovine serum albumin, $\mathrm{pH} 7 \cdot 4$ ) containing $2 \mathrm{mM} \mathrm{CaCl}_{2}$ (TH-Ca). Incubations in all of the experiments were carried out at room temperature unless specified otherwise. Cells were counted electronically with a System 9000 Hematology Series cell counter (Serono Baker Diagnostics, Allentown, Pennsylvania, USA).

\section{ISOLATION OF PMN}

CHEMOTACTIC SEPARATION OF PMN

PMN were diluted to $2 \times 10^{6} / \mathrm{ml}$ in Gey's balanced salt solution (GBSS) (Life Technologies) 
containing $0 \cdot 2 \%$ bovine serum albumin. Migrated and non-migrated PMN subpopulations were separated in chemotaxis separation chambers (Neuro Probe, Cabin John, Maryland, USA) containing $10^{-7} \mathrm{M} \mathrm{N}$-formylmethionylleucyl-phenylalanine (FMLP) (Sigma, St Louis, Missouri, USA) in the lower wells and a polyvinylpyrrolidone-free polycarbonate membrane filter $(5 \mu \mathrm{m}$ pores; Poretics, Livermore, California, USA) as described previously. ${ }^{89}$ Control cells were held in suspension at $4^{\circ} \mathrm{C}$ (unstimulated) or at $37^{\circ} \mathrm{C}$ with $10^{-7}$ M FMLP (stimulated) for 35 minutes.

\section{ANTIBODIES}

The fluorescein isothiocyanate (FITC) conjugated CD41 monoclonal antibody (clone P2; Immunotech, Westbrook, Maine, USA) recognises an epitope on glycoprotein GPIIb only present on the platelet membrane GPIIb-IIIa complex. The phycoerythrin (PE) conjugated CD62P monoclonal antibody (clone AC1.2; Becton Dickinson Immunocytometry Systems, San Jose, California, USA) recognises the CD62P (P-selectin) antigen, an $\alpha$-granule membrane protein that is exported and expressed on the plasma membrane of activated platelets. The PE conjugated CD45 monoclonal antibody (clone KC56(T-200); Coulter, Hialeah, Florida, USA) recognises the leucocyte common antigen. The isotype control reagents conjugated to either FITC or PE were purchased from Becton Dickinson Immunocytometry Systems.

\section{FLUORESCENT LABELLING OF PLATELETS AND PMN}

Platelets in plasma were labelled with monoclonal antibody as described previously. ${ }^{12}$ Platelets in TH-Ca were incubated for 30 minutes with saturating concentrations of FITC conjugated CD41 (GPIIb-IIIa) or PE conjugated CD62P (P-selectin) monoclonal antibody. The samples were fixed with an equal volume of $2 \%$ formaldehyde for 30 minutes, washed once and resuspended in TH-Ca. Platelets were suspended at a density of $3 \times 10^{8} / \mathrm{ml}$ for co-incubation assays and at $10^{7} / \mathrm{ml}$ for platelet assays. For activation of platelets, $\alpha$-thrombin (1.0 unit/ml; lot H2; FDA, Bethesda, Maryland, USA) was added for 10 minutes before the addition of fixative. PMN preparations were labelled using PE conjugated CD45 monoclonal antibody as a PMN marker and FITC conjugated CD41 monoclonal antibody as a marker for contaminating platelets. The samples were incubated at $4^{\circ} \mathrm{C}$ for 30 minutes, washed and resuspended at a density of $3 \times 10^{6} \%$ $\mathrm{ml}$ in GBSS.

\section{CO-INCUBATION ASSAY}

Fixed, labelled platelets $\left(150 \mu \mathrm{l}\right.$ of $\left.3 \times 10^{8} / \mathrm{ml}\right)$ were added to viable, labelled PMN $(150 \mu \mathrm{l}$ of $3 \times 10^{6} / \mathrm{ml}$ ) to approximate the relative densities in normal blood. The samples were mixed by gentle inversion, incubated for 30 minutes at $4^{\circ} \mathrm{C}$, and analysed by flow cytometry. For the divalent cation depletion study, $2 \mathrm{mM}$ EDTA (Sigma) was added to each cell fraction for 20 minutes before co-incubation.

\section{FLOW CYTOMETRY}

A FACScan flow cytometer (Becton Dickinson) was used to acquire light scatter and fluorescence signals for at least 10000 events from each sample. Gating on platelet events and PMN events was performed using forward and side scatter, with FITC conjugated CD41 monoclonal antibody as a platelet specific marker, and PE conjugated CD45 monoclonal antibody as a PMN specific marker. Results are expressed as histograms of cell count versus log fluorescence intensity (FL1-H). Non-specific binding, obtained from parallel assays with FITC or PE labelled isotype control monoclonal antibody, was used to set a threshold marker for positive platelet fluorescence. The marker excluded $99 \%$ of the isotype control fluorescence. The percentage of activated platelets in the platelet preparations was determined by the proportion of FITC conjugated CD41 positive particles also expressing greater than background levels of CD62P (P-selectin, activation dependent surface antigen released from $\alpha$-granules) estimated using the PE conjugated CD62P monoclonal antibody. The platelet fluorescence ratio, an estimate of the number of platelets bound to each PMN, was determined as described previously, ${ }^{7}$ from the ratio of the mean platelet marker fluorescence in the PMN gate to the mean platelet marker fluorescence in the platelet gate. The experiments were performed at least four times with a single representative experiment shown in figs 1 and 3, and a summary graph shown in fig 2 .

\section{FLUORESCENCE MICROSCOPY}

All samples were stained with FITC conjugated CD41 and PE conjugated CD45 monoclonal antibodies, fixed in suspension with $1 \%$ formaldehyde, washed and cytocentrifuged onto microscope slides. Unbound platelets were washed out of the co-incubation samples prior to fixation. Slides were mounted in Gel-mount medium (Biomeda, Foster City, California, USA). Cells were visualised using a Nikon Microphot-FX microscope. Images were acquired using a $\times 40$ DIC Nikon objective lens (numerical aperture 0.70).

\section{STATISTICAL ANALYSIS}

Percentages of PMN subpopulations with adhered platelets were analysed using a paired $t$ test. Differences were considered significant if $\mathrm{p}<0.005$.

\section{Results}

ADHESION OF CONTAMINATING PLATELETS TO MIGRATED AND NON-MIGRATED PMN

Isolated PMN were stimulated with the chemoattractant FMLP and migrated and nonmigrated PMN subpopulations were separated. 

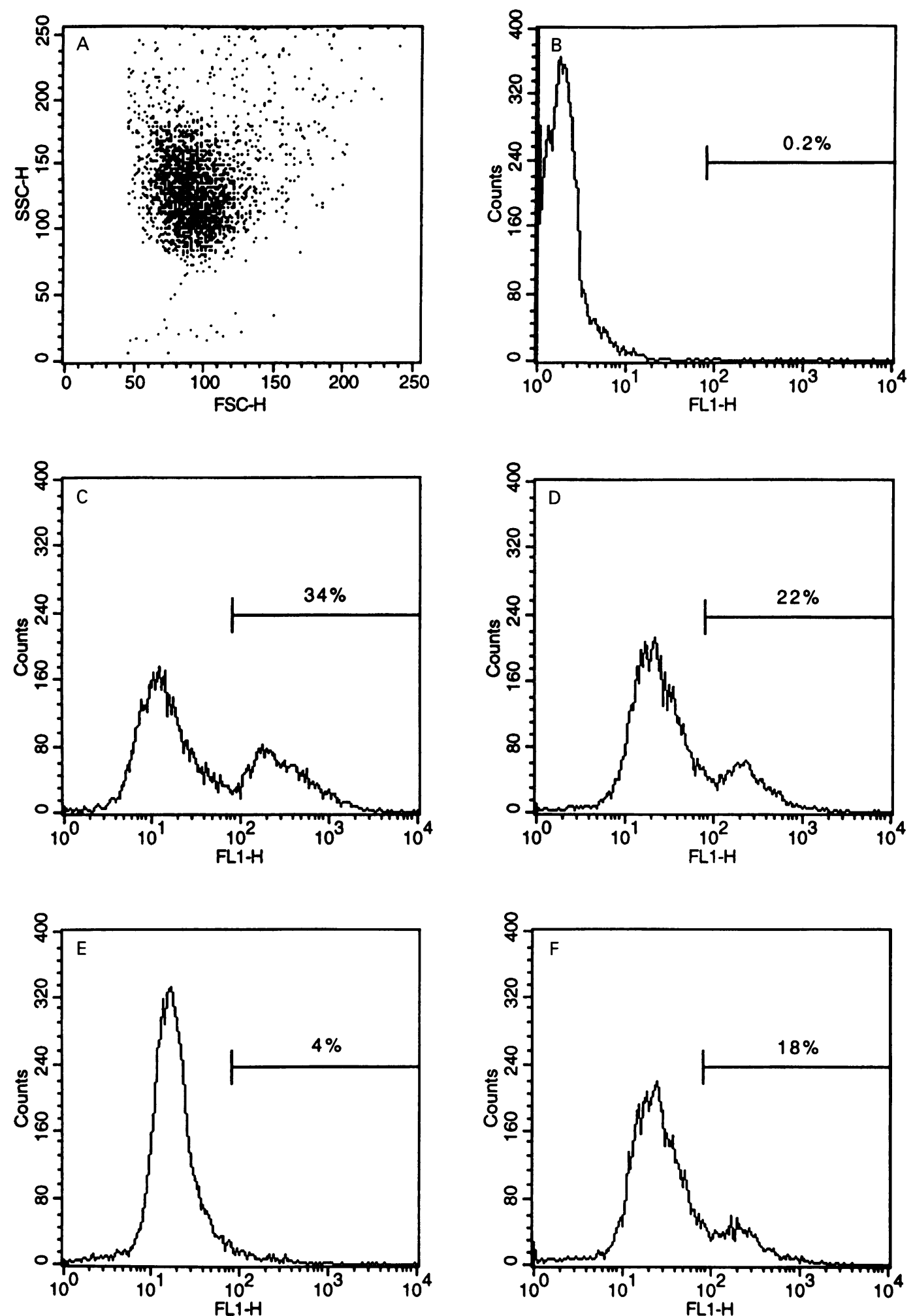

Figure 1 Flow cytometric analysis of platelet-PMN adhesion in migrated and non-migrated subpopulations of PMN. Isolated PMN stimulated with $10^{-7}$ M FMLP were separated in a chemotaxis separation chamber. The migrated and non-migrated PMN subpopulations, adjusted to a cell density of $3 \times 10^{6} / \mathrm{ml}$ in GBSS, were labelled with platelet specific FITC conjugated CD41 monoclonal antibody or an isotype control monoclonal antibody. (A) Dot plot of side scatter (SSC) versus forward scatter (FSC), showing PMN events and excluding single platelets. (B) Histogram plot of an isotype matched control monoclonal antibody (mouse IgG1-FITC) labelled PMN preparation. FL1-H (FITC) was used to set the threshold marker for positive platelet fluorescence. (C) and (D) correspond to fluorescence of platelets attached to unstimulated and stimulated PMN controls in suspension. $(E)$ and $(F)$ correspond to fluorescence of platelets attached to migrated and non-migrated PMN. The number over each marker represents the percentage of PMN binding platelets.

Flow cytometric analysis of contaminating platelets, labelled with FITC conjugated CD41, adhering to isolated PMN is shown in fig 1. PMN events (fig 1A), positive for FITC conjugated CD41, represent PMN with bound platelets. The marker shown in figs $1 \mathrm{~B}-1 \mathrm{~F}$ is set such that it excludes $99 \%$ of the isotype control (fig 1B). Events included in this marker represent PMN bound platelets.

In the suspended PMN controls (figs $1 \mathrm{C}$ and 1D) $34 \%$ of unstimulated PMN bound platelets and $22 \%$ of FMLP stimulated PMN 


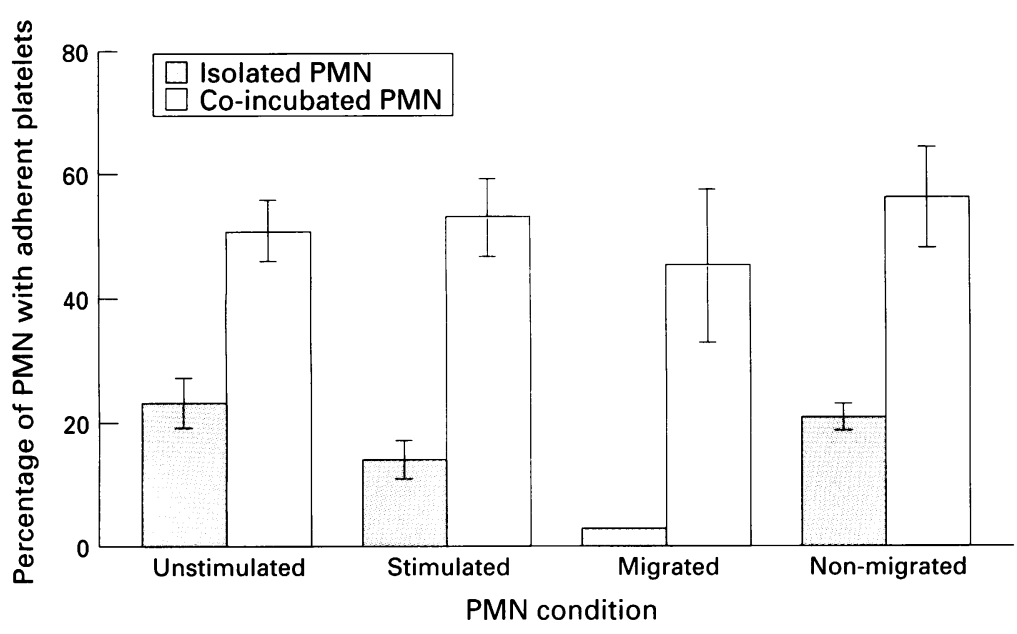

Figure 2 Migrated and non-migrated PMN adhesion to unactivated platelets. The data presented are mean percentage values of four separate experiments performed with different donors \pm 1 SEM. The percentage of PMN with attached platelets was determined from flow cytometric analysis of platelet marker fluorescence in the PMN gate. Shaded bars represent heterotypic adhesion in isolated PMN and open bars represent heterotypic adhesion in co-incubation assays. some PMN, with one platelet bound per PMN (figs $4 \mathrm{~A}$ and $4 \mathrm{~B}$ ). When PMN were co-incubated with platelets, an increase in the number of PMN with bound platelets was observed (figs 4C and 4D). One or two platelets were bound to each PMN. A control consisting of PMN co-incubated with thrombin activated platelets (figs $4 \mathrm{E}$ and $4 \mathrm{~F}$ ) showed an increased number of PMN with bound platelets and an increased number of platelets bound to each PMN.

\section{Discussion}

Platelet-PMN interactions occur as part of normal cell functions during inflammatory and haemostatic processes. We have used flow cytometry to study the adhesion of unactivated platelets to migrated and non-migrated PMN. In isolated PMN preparations a significant percentage of PMN (23\%) bound contaminating, unactivated platelets. This was confirmed by fluorescence microscopy. EDTA did not alter platelet adherence, indicating that the binding was independent of divalent cations. Previous studies have reported the absence or very low (less than 3\%) platelet contamination in isolated PMN. ${ }^{71314}$ Sporadic observations of a second phase calcium response in stimulated PMN have been attributed to contaminating platelets. ${ }^{15-17}$ Chabannes et al ${ }^{18}$ reported contamination of PMN by platelets, measured by phase contrast microscopy, to be $0 \cdot 5-1$ platelet per PMN. Our study has demonstrated that a subset of isolated PMN binds platelets. When the PMN were separated into migrated and non-migrated subpopulations, the bound platelets became detached from the migrated PMN during chemotaxis (fig 1). We have shown previously that adhesion molecules, including CD11a, CD11b, CD11c, CD31, CD43, and CD44, are downregulated differentially after stimulation of PMN by FMLP. ${ }^{10}$ These adhesion molecules may be involved in regulating receptor-ligand interaction between the platelets and migrated PMN.

When PMN were co-incubated with unactivated platelets in a ratio approximating the relative concentrations in normal blood, there was a twofold increase in the percentage of PMN with bound platelets compared with isolated PMN alone. About $50 \%$ of the PMN coincubated with platelets bound platelets. This adherence could not be attributed to the small number of platelets $(7 \%)$ expressing low levels of $\mathrm{CD} 62 \mathrm{P}$ in the unactivated platelet preparation. The percentages of migrated and nonmigrated PMN with bound platelets after coincubation were similar. This adherence occurred in the absence of divalent cations.

The adherence of platelets to unactivated and activated PMN differs, the latter being mediated by platelet CD62P (P-selectin) and PMN P-selectin glycoprotein ligand-1. The percentage of PMN with attached, unactivated platelets in the present study is higher than that reported previously. ${ }^{71920}$ Contact dependent interaction between PMN and platelets amplifies chemotactic peptide induced changes in PMN filamentous actin content, intracellular calcium
FLUORESCENCE MICROSCOPY

Platelet-PMN interactions examined by microscopy are shown in fig 4 . In the isolated PMN suspension, contaminating platelets adhered to 

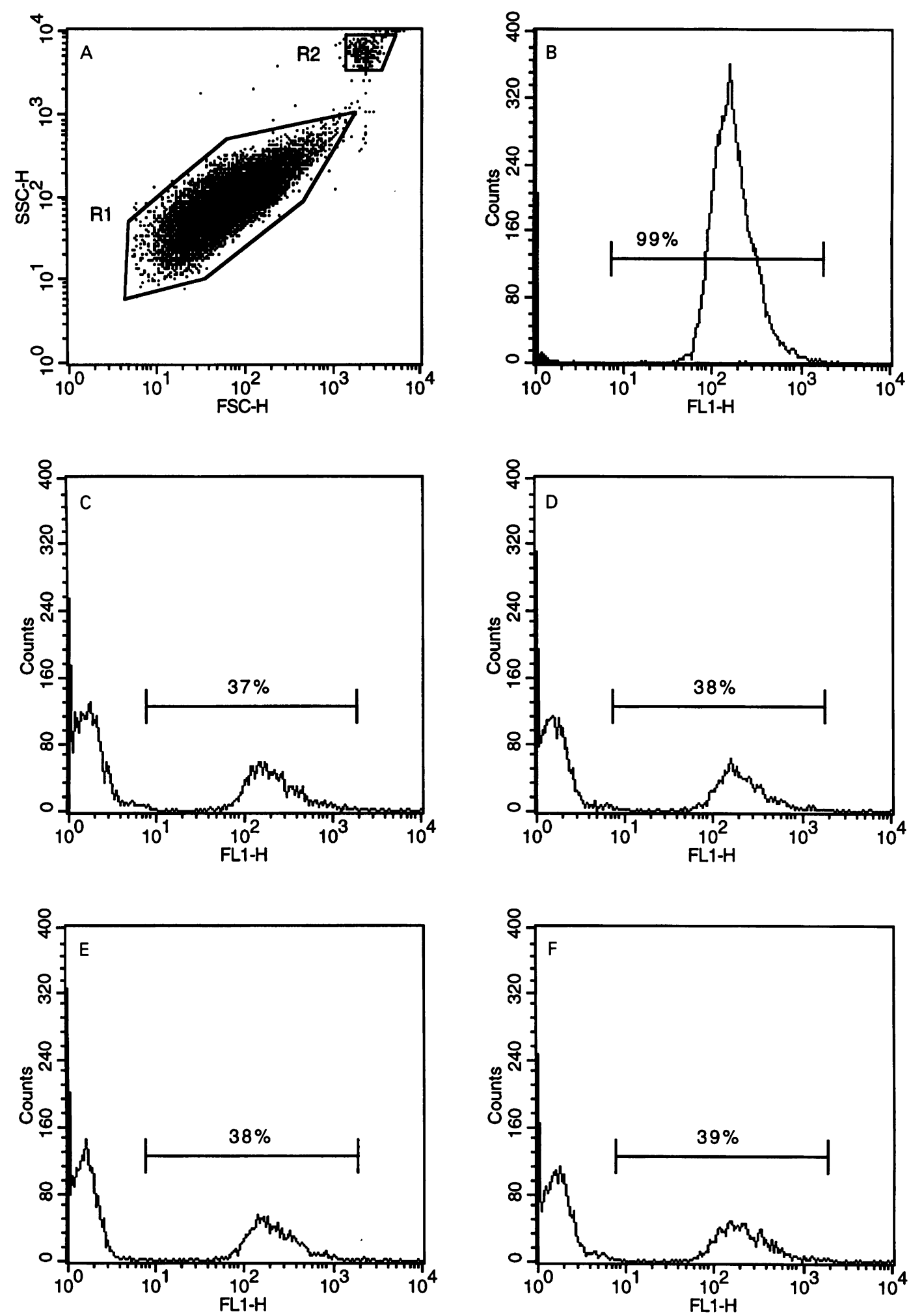

Figure 3 Effect of co-incubating unactivated platelets with migrated and non-migrated PMN on platelet-PMN adherence. Unactivated platelets in TH were labelled with FITC conjugated CD41 monoclonal antibody, fixed with $2 \%$ formaldehyde, washed, and resuspended in TH. Migrated and non-migrated subpopulations of PMN were separated after stimulation with $10^{-7} M F M L P$, using a chemotaxis separation chamber. Platelets were co-incubated with $P M N$ (final density of $1.5 \times 10^{8}$ platelets $/ \mathrm{ml}$ and $1.5 \times 10^{10} \mathrm{PMN} / \mathrm{ml}$ ) for 30 minutes at $4^{\circ} \mathrm{C}$. (A) Dot plot of side scatter (SSC) versus forward scatter (FSC) with platelet events gated in R1 and PMN events gated in R2. (B) Histogram of FITC conjugated CD41 (FL1-H) expression on platelets in the platelet gate R1 (open histogram). An isotype control (mouse IgG1-FITC; filled histogram) was used to set the threshold marker for positive platelet fluorescence. The marker excluded $99 \%$ of the isotype control fluorescence. $(C)$ to $(F)$ represent FITC conjugated CD41 fluorescence in the PMN gate (R2). (C) Platelets co-incubated with control, unstimulated PMN in suspension. (D) Platelets co-incubated with control, stimulated PMN in suspension. (E) Co-incubation of platelets with migrated PMN. (F) Co-incubation of platelets with non-migrated PMN. The number over each marker represents the percentage of PMN binding platelets.

concentration, and possibly G-protein mediated signal transduction. ${ }^{17}$ Further studies on the cytoskeletal changes and regulation of adhesion molecules in platelet-PMN in- teractions are required. The present study has characterised the adherence of unactivated platelets to migrated and non-migrated PMN. The specific receptor-ligand interactions in this 

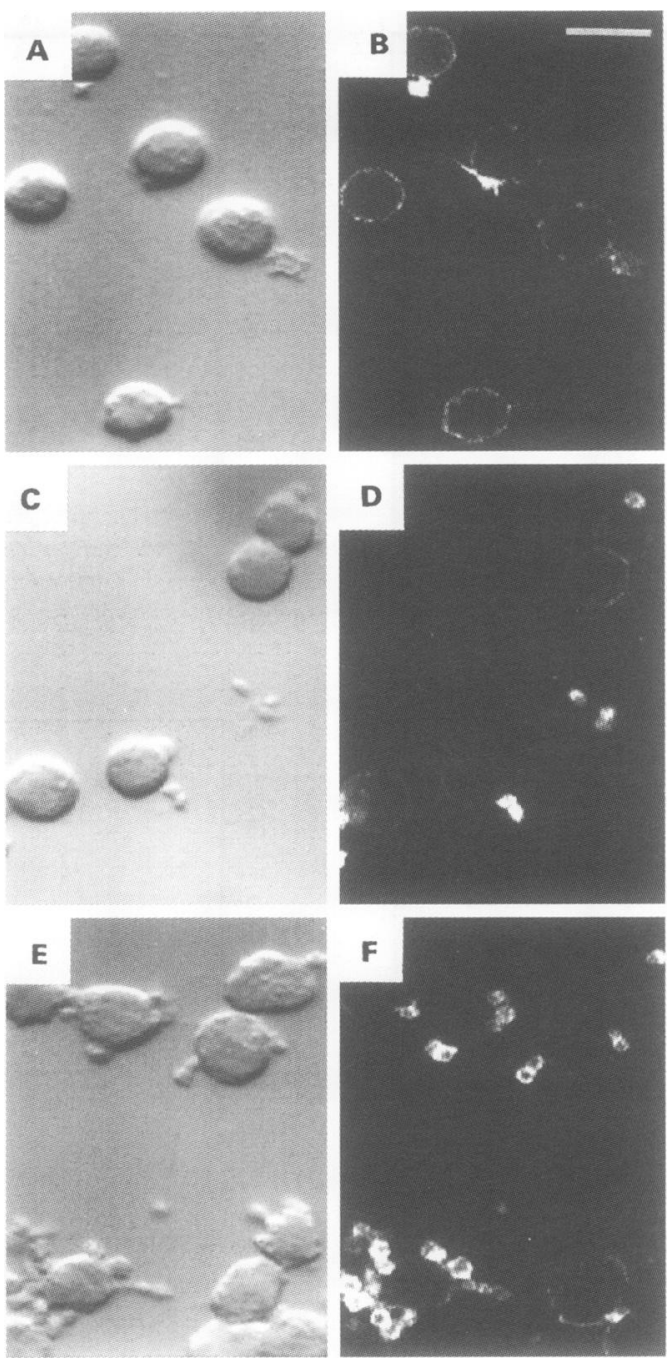

Figure 4 PMN-platelet adhesion. Platelet fluorescence was visualised with FITC conjugated CD41 monoclonal antibody and PMN fluorescence with $P E$ conjugated $C D 45$ monoclonal antibody. $(A),(C)$ and $(E)$ Phase Nomarski images. (B), (D) and (F) FITC conjugated $C D 41 / P E$ conjugated CD45 fluorescence images, excited at $488 \mathrm{~nm}$ and visualised with a $550 \mathrm{~nm}$ barrier filter. The bar represents $10 \mu \mathrm{m}$. (A) and (B) Isolated PMN with contaminating platelets. (C) and (D) Co-incubated PMN and unactivated platelets. (E) and $(F)$ Co-incubated $P M N$ and activated platelets.

heterotypic cell adhesion and their relevance in inflammation and haemostasis remain to be elucidated.
1 Faint RW. Platelet-neutrophil interactions: their significance, Blood Rev 1992;6:83-91.

2 Weksler BB. Platelets. In: Gallin JI, Goldstein IM, Snyderman R, eds. Inflammation: basic principles and clinical correlates. New York: Raven Press, 1992:727-46.

3 Larsen E, Celi A, Gilbert GE, Furie BC, Erban JK, Bonfanti $\mathrm{R}$, et al. PADGEM protein: a receptor that mediates the interaction of activated platelets with neutrophils and monocytes. Cell 1989;59:305-12.

4 Hamburger SA, McEver RP. GMP-140 mediates adhesion of stimulated platelets to neutrophils. Blood 1990;75:550-4.

5 Bevilacqua M, Butcher E, Furie B, Furie B, Gallatin M, Gimbrone $M$, et al. Selectins: a family of adhesion receptors [letter]. Cell 1991;67:233.

6 Asa D, Raycroft L, Ma L, Aeed PA, Kaytes PS, Elhammer $\mathrm{AP}$, et al. The P-selectin functions as a common human leukocyte ligand for P-and E-selectins. 7 Biol Chem 1995; 270:11662-70.

7 Rinder HM, Bonan JL, Rinder CS, Ault KA, Smith BR. Activated and unactivated platelet adhesion to monocytes and neutrophils. Blood 1991;78:1760-9.

8 Harvath L, Leonard EJ. Two neutrophil populations in human blood with different chemotactic activities: separation and chemoattractant binding. Infect Immun 1982; 36:443-9.

9 Falk W, Harvath L, Leonard EJ. Only the chemotactic subpopulation of human blood monocytes expresses receptors for the chemotactic peptide $\mathrm{N}$-formyl-methionylleucyl-phenylalanine. Infect Immun 1982;36:450-4.

10 Harvath L, Brownson NE, Terle DA. Downregulation of human neutrophil adhesion molecule surface expression after chemotaxis. In: Schlossman SF, Boumsell L, Gilks W, Harlan JM, Kishimoto T, Morimoto C, et al, eds. Leukocyte typing $\mathrm{V}$ : white cell differentiation antigens. Proceedings of the Fifth Intermational Workshop and Conference; 1993 Nov 3-7; Boston (MA). New York (NY): ference; 1993 Nov 3-7; Boston (MA).

11 Harvath L, Balke JA, Christiansen NP, Russell AA, Skubitz KM. Selected antibodies to leukocyte common antigen (CD45) inhibit human neutrophil chemotaxis. 7 Immunol 1991;146:949-57.

12 Pastakia KB, Terle D, Prodouz KN. Penicillin-induced dysfunction of platelet membrane glycoproteins. $\mathcal{F} \mathrm{Lab}$ Clin Med 1993;121:546-54.

13 Ruf A, Schlenk RF, Maras A, Morgenstern E, Patscheke $\mathrm{H}$. Contact-induced neutrophil activation by platelets in human cell suspensions and whole blood. Blood 1992;80: 1238-46.

14 Rinder HM, Tracey JL, Rinder CS, Leitenberg D, Smith BR. Neutrophil but not monocyte activation inhibits Pselectin-mediated platelet adhesion. Thromb Haemost 1994;71:750-6.

15 Chandler DE, Kazilek CJ. Calcium signals in neutrophils can be divided into three distinct phases. Biochim Biophys Acta 1987;931:175

16 Sage SO, Pintado E, Mahaut-Smith MP, Merritt JE. Rapid kinetics of agonist-evoked changes in cytosolic free $\mathrm{Ca}^{2}$. concentration in fura-2-loaded human neutrophils. Biochem $\mathcal{F}$ 1990;265:915-8.

17 Bengtsson T, Grenegard M. Platelets amplify chemotactic peptide-induced changes in $\mathrm{F}$-actin and calcium in human peptide-induced changes in F-actin and calciun

18 Chabannes B, Moliere P, Pacheco Y, Lagarde M. Decreased arachidonic acid metabolism in human platelets by autologous neutrophils: possible role of cell adhesion. Biochem f 1994;300:685-91.

19 Jungi TW, Spycher MO, Nydegger UE, Barandun S. Platelet-leukocyte interaction: selective binding of thrombinstimulated platelets to human monocytes, polymorphonuclear leukocytes and related cell lines. Blood morphonuclear

20 de Bruiine-Admiraal LG, Modderman PW, Von dem Borne AEGKr, Sonnenberg A. P-selectin mediates calcium-dependent adhesion of activated platelets to many different
types of leukocytes: detection by flow cytometry. Blood 1992;80: $134-42$. 